\title{
Energy Reconstruction with the Radio Neutrino Observatory Greenland (RNO-G)
}

Christoph Welling ${ }^{a, *}$ on behalf of the RNO-G Collaboration

(a complete list of authors can be found at the end of the proceedings)

${ }^{a}$ Deutsches Elektronen-Synchrotron (DESY),

Platanenallee 6, 15738 Zeuthen, Germany

E-mail: christoph.welling@desy.de

Starting in summer 2021, the Radio Neutrino Observatory Greenland (RNO-G) will attempt to achieve the first detection of neutrinos with energies at the $\mathrm{EeV}$ scale. We present a method to reconstruct the energy of neutrino-induced particle showers detected by RNO-G and the resolution on the neutrino energy this would allow. We demonstrate that to further improve the neutrino energy reconstruction, a way to identify the neutrino flavor is needed.

$37^{\text {th }}$ International Cosmic Ray Conference (ICRC 2021)

July 12th-23rd, 2021

Online - Berlin, Germany

\footnotetext{
*Presenter
} 


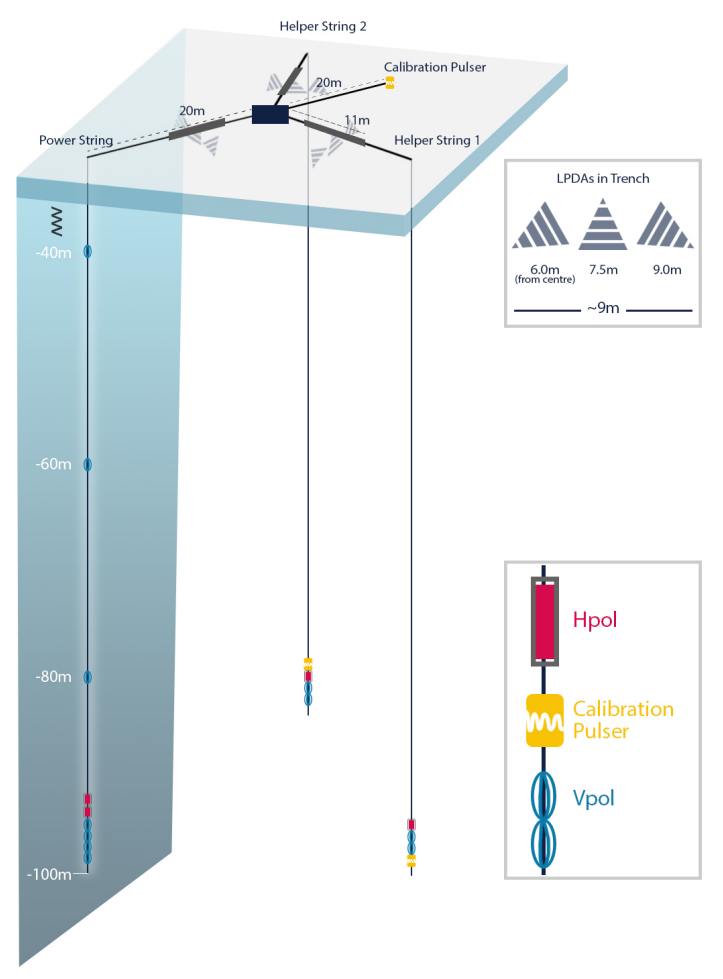

Figure 1: Drawing of one of the 35 stations that will be part of the Radio Neutrino Observatory Greenland (RNO-G).

\section{Introduction}

The Radio Neutrino Observatory Greenland (RNO-G) [1] will consist of 35 detector stations arranged on a grid with a spacing of $1.25 \mathrm{~km}$ near Summit Station on the top of the Greenland ice sheet. By detecting radio signals from neutrino-induced particle showers in the ice over distances of multiple kilometers, it will achieve a detection volume of around $100 \mathrm{~km}^{3}$, which is needed to access the neutrino flux at energies above $\sim 10 \mathrm{PeV}$. The radio antennas are deployed in three holes up to a depth of $100 \mathrm{~m}$ and buried just below the snow surface. From the signals they detect, the shower energy can be reconstructed, but determining the energy of the neutrino is complicated by the fact that only an unknown fraction of the neutrino energy is transferred into the shower.

\section{Reconstruction of the neutrino energy}

RNO-G detects the radio emission stemming from particle cascades that follow neutrino interactions $[2-5,5]$. When a neutrino interacts with an atom in the ice, it transfers a fraction $y$ of its energy to the atomic nucleus. This fraction is random and cannot be measured, so it puts a limit on our ability to reconstruct the neutrino energy. In this section, we discuss these lower limits and show how they affect the achievable neutrino energy resolution.

Using Bayes' theorem, we can calculate the probability density of $\lg \left(E_{v}\right)$ given a reconstructed 
shower energy $\lg \left(E_{r}\right)$ :

$$
p\left(\lg \left(E_{v}\right) \mid \lg \left(E_{r}\right)\right)=\frac{p\left(\lg \left(E_{r}\right) \mid \lg \left(E_{v}\right)\right) \cdot p\left(\lg \left(E_{v}\right)\right)}{p\left(\lg \left(E_{r}\right)\right)}
$$

Because the energies cover several orders of magnitude, it is practical to use the base 10 logarithm of the energy.

\subsection{Neutrino Energy Spectrum}

The $p\left(\lg \left(E_{v}\right)\right)$ term in Eq.1 describes the energy distribution of neutrinos interacting inside the detector volume. For a given spectrum $S$, this distribution is

$$
p\left(\lg \left(E_{v}\right) \mid S\right)=\frac{\Phi_{\nu}\left(E_{v}\right) / \lambda_{v}\left(E_{v}\right) \cdot E_{v}}{\int d \lg \left(E_{v}\right) \Phi_{\nu}\left(E_{v}\right) / \lambda_{\nu}\left(E_{v}\right) \cdot E_{v}}
$$

Where $\Phi_{v}$ is the neutrino flux and $\lambda_{v}$ the interaction length of the neutrino. Unfortunately, we do not yet know the neutrino flux at the energies accessible by RNO-G, so we consider a set of spectra that each have a certain probability of being true. We assume all spectra follow a power law $\Phi_{v}\left(E_{v}\right) \propto E_{v}^{-\gamma}$, whose spectral index we do not know. If we assign a prior probability $p(\gamma)$ to each spectrum, the expected neutrino energy distribution is

$$
p\left(\lg \left(E_{v}\right)\right)=\frac{\int d \gamma \quad p(\gamma) \cdot E_{\nu}^{-\gamma+1} / \lambda_{\nu}\left(E_{v}\right)}{\iint d \gamma d E_{v} p(\gamma) \cdot E_{\nu}^{-\gamma+1} / \lambda_{\nu}\left(E_{v}\right)}
$$

Assigning these probabilities to the spectra is of course very subjective without having measured a flux. For this study, we choose $\gamma$ to follow a uniform distribution in the range $2<\gamma<3$, but in principle any probability is possible.

\subsection{Interaction Dynamics}

When the neutrino interacts in the ice, only a fraction $\kappa$ of its energy is transferred into a shower. If it has a flavor other than $v_{e}$ or interacts via neutral current interactions, this fraction is given by the interaction inelasticity $[6,7]$. If it is an electron neutrino and interacts via a charged current interaction, an electromagnetic shower is also produced. If the energy of the electromagnetic shower is small enough for it to not be affected by the LPM effect, both showers overlap and effectively $\kappa=1$. At higher energies, the LPM effect can cause the showers to separate and stretching the electromagnetic shower into consisting of several smaller sub-showers. Unfortunately, the interference of the radio signals from the two showers is too complex to be included in this model, however if both showers can be detected and isolated separately, it may allow for the inelasticity of the neutrino interaction to be measured. If there is a probability $P(h)$ for the neutrino interaction to only produce a hadronic shower, and $P(e)=1-P(h)$ to produce a hadronic and an electromagnetic shower, both cases can be combined to

$$
p\left(\lg \left(E_{\nu}\right)\right)=p\left(\lg \left(E_{\nu}\right) \mid h\right) \cdot P(h)+p\left(\lg \left(E_{\nu}\right) \mid e\right) \cdot P(e)
$$



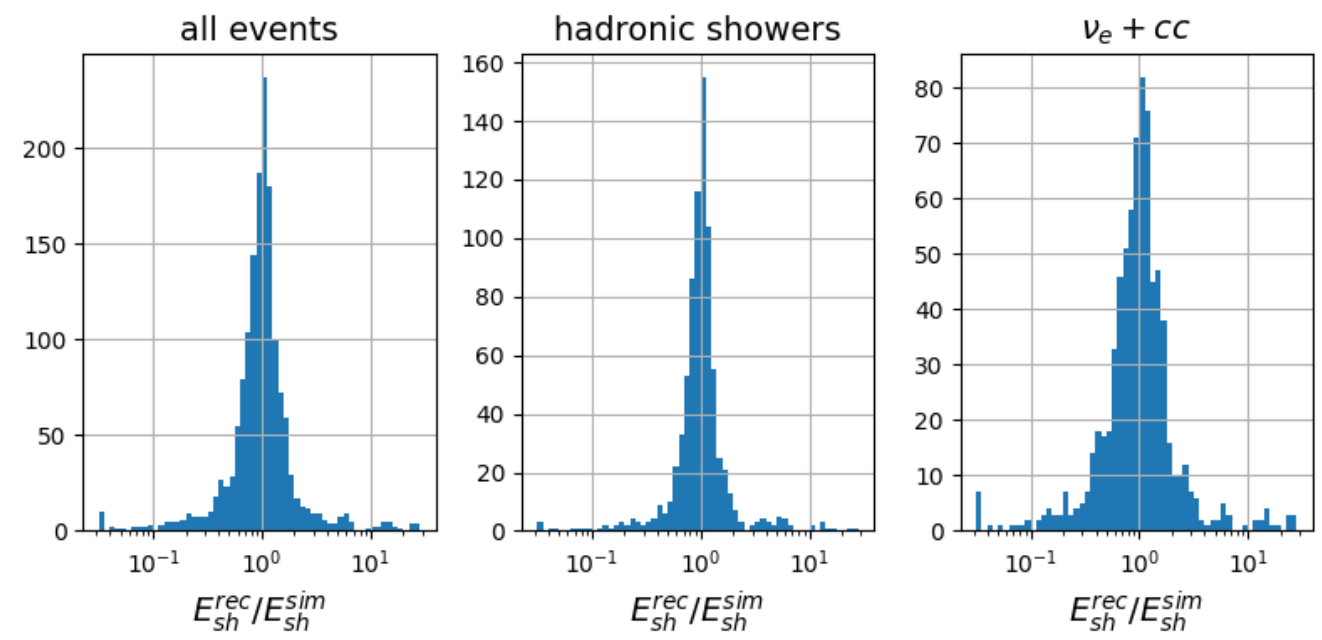

Figure 2: Histogram of the ratio between reconstructed and actual shower energy for all events (left), those that only produce a hadronic shower (middle) and those where electron neutrinos undergo a charged current interaction (right).

So far, no quantitative study has been conducted on how to identify the neutrino flavor with radio detectors, so we assume that the probability that a given event produces a hadronic and an electromagnetic shower is $P(e)=\frac{1}{3} \cdot 0.71$, the probability of the flavor being $v_{e}$, assuming equal mixing, times the probability for it to undergo charged-current interactions [8].

While this describes the energy distribution of showers produced in the detection volume, showers with higher energies have a much better chance of being detected. To account for this, we need to include the probability $p_{T}\left(\lg \left(E_{S}\right)\right)$ for a shower with a given energy to be detected:

$$
p\left(\lg \left(E_{S}\right) \mid \lg \left(E_{v}\right)\right)=p_{T}\left(\lg \left(E_{S}\right)\right) \cdot p\left(\lg \left(E_{S} / E_{v}\right)\right)
$$

which shifts the energy distribution towards events with a higher $E_{S} / E_{\nu}$.

\subsection{Shower Energy Reconstruction}

We have developed a method to reconstruct the shower energy by first reconstructing the position of the neutrino interaction using the arrival times of the radio signal at different antennas. Then the radio signal is reconstructed using a novel method based on Information Field Theory. After the spectrum of the radio signal has been corrected for propagation effects, its shape and magnitude are used as an estimator for the shower energy. More details of this method are described it $[9,10]$.

The resulting energy resolution is shown in Fig.2. Events where only a hadronic shower is created have a better energy resolution because interference between the radio signals from the hadronic and the electromagnetic shower do not obey all assumptions made for the reconstruction method. The shower energy resolution is also energy-dependent, but we simplify this by assuming the same resolution for all energies and both event types. Over all events, the $68 \%$ quantile of the $\lg \left(E_{s}^{r e c} / E_{S}^{s i m}\right)$ distribution is $[-0.13,0.12]$. 


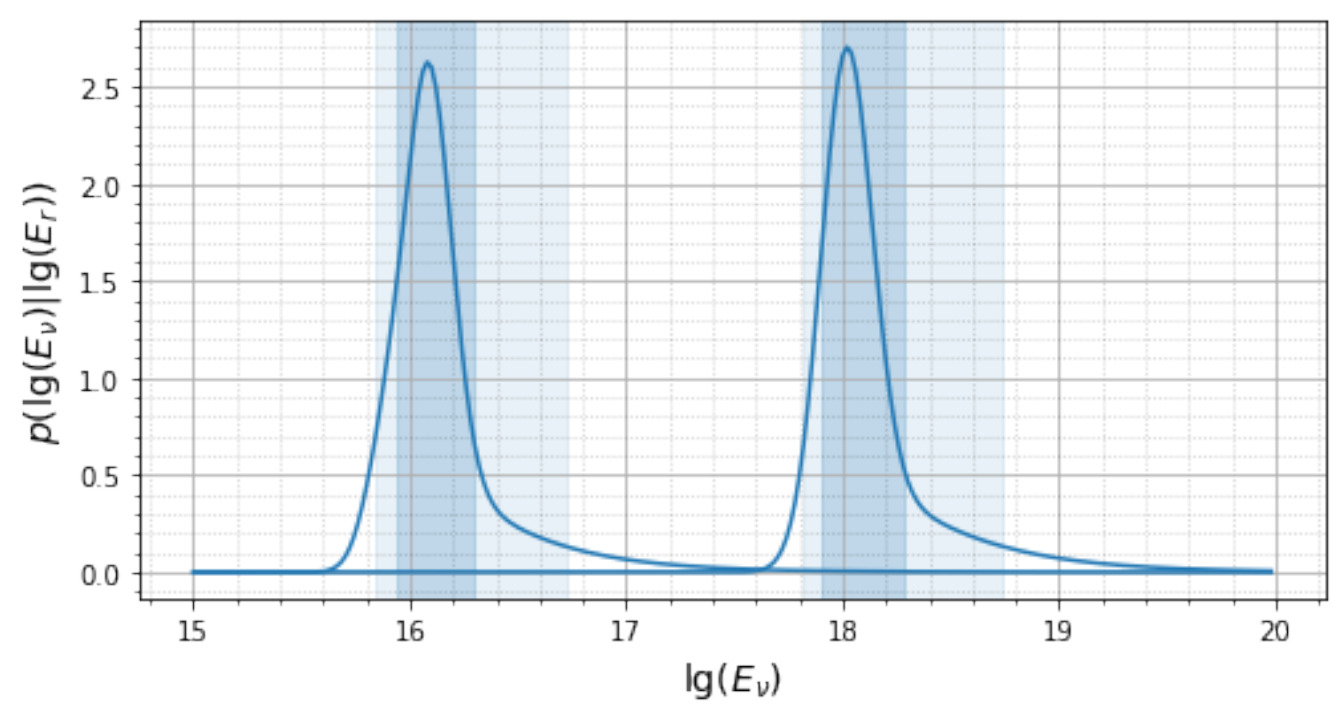

Figure 3: Posterior distributions of the neutrino energy for reconstructed shower energies of $1 \times 10^{16} \mathrm{eV}$ and $1 \times 10^{18} \mathrm{eV}$. The shaded regions mark the $68 \%$ and $90 \%$ quantiles.

We approximate the uncertainties on the reconstructed shower energies by a Gaussian distribution $\mathcal{N}\left(\lg \left(E_{r} / E_{S}\right) \mid 0, \sigma_{\lg (E)}\right)$ with the standard deviation $\sigma_{\lg (E)}$. Then the posterior for obtaining a reconstructed shower energy $E_{r}$ from a neutrino with energy $E_{v}$ is given by

$$
p\left(\lg \left(E_{r}\right) \mid \lg \left(E_{v}\right)\right)=\int d \lg \left(E_{s}\right) \quad \mathcal{N}\left(\lg \left(E_{r} / E_{s}\right) \mid 0, \sigma_{\lg (E)}\right) \cdot p\left(\lg \left(E_{s}\right) \mid \lg \left(E_{v}\right)\right)
$$

With this, the posterior probability on the neutrino energy for a given reconstructed shower energy can be calculated from Eq. 1. The denominator $p\left(\lg \left(E_{r}\right)\right)$ is the integral of the numerator over $\lg \left(E_{v}\right)$, so all that is needed is to make sure the result is properly normalized. The result is shown in Fig. 3 for shower energies of $1.0 \times 10^{16} \mathrm{eV}$ and $1.0 \times 10^{18} \mathrm{eV}$. While the distributions are peaked around the reconstructed shower energy being equal to the neutrino energy, they also have a tail towards higher energies.

\section{Influences on the Neutrino Energy Resolution}

Section 2 uses some assumptions to obtain the neutrino energy resolution. We discuss their influences in the following.

\subsection{Spectrum}

The posterior probability $p\left(\lg \left(E_{v}\right) \mid \lg \left(E_{r}\right)\right)$ for a given neutrino energy is directly proportional to its prior probability $p\left(\lg \left(E_{v}\right)\right)$, as shown in Eq. 1, so the chosen spectrum model will have a direct effect on neutrino energy reconstruction. We demonstrate this by choosing a much softer set of spectra, with the spectral index uniformly distributed in the range $3<\gamma<4$. The effect this has on the neutrino energy posterior is shown in Fig. 4. Unsurprisingly, the neutrino energies are shifted slightly towards lower energies. The tails of the distributions are much smaller, which also 


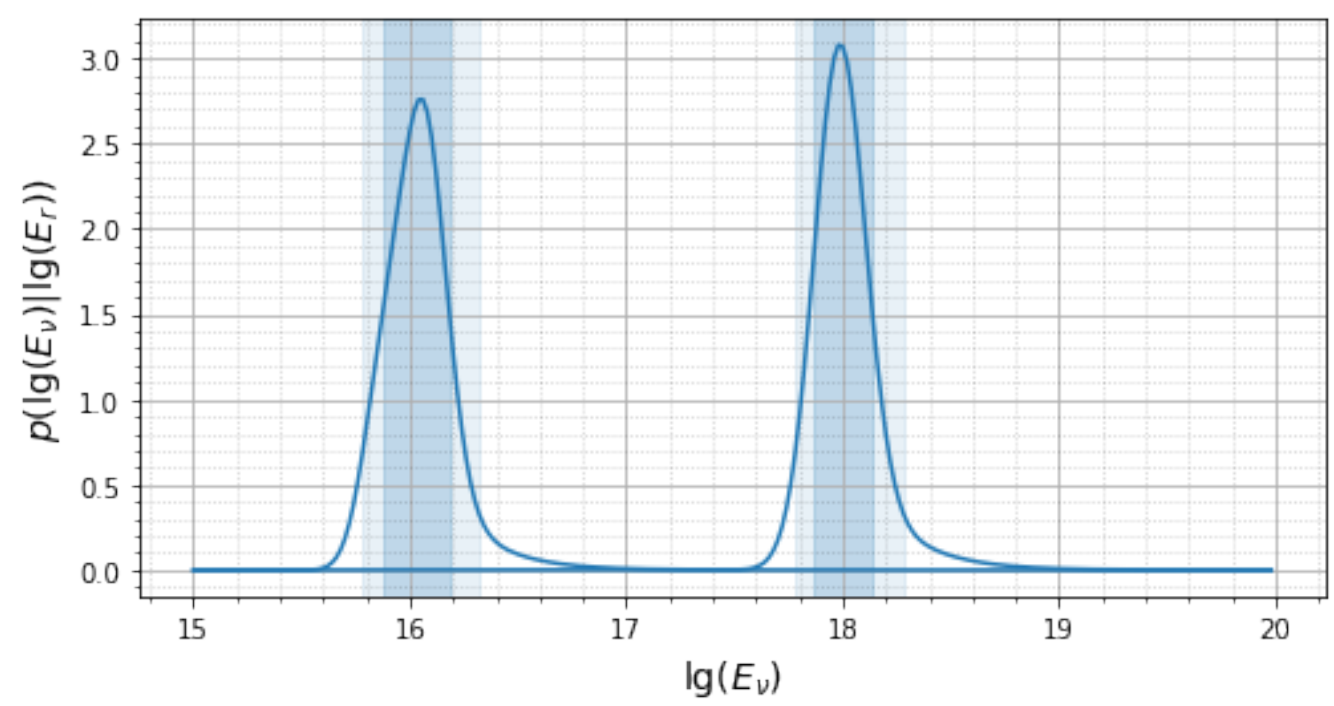

Figure 4: Posterior distributions of the neutrino energy for reconstructed shower energies of $1 \times 10^{16} \mathrm{eV}$ and $1 \times 10^{18} \mathrm{eV}$, if a spectrum model with spectral indices in the range $3<\gamma<4$ is assumed. The shaded regions mark the $68 \%$ and $90 \%$ quantiles.

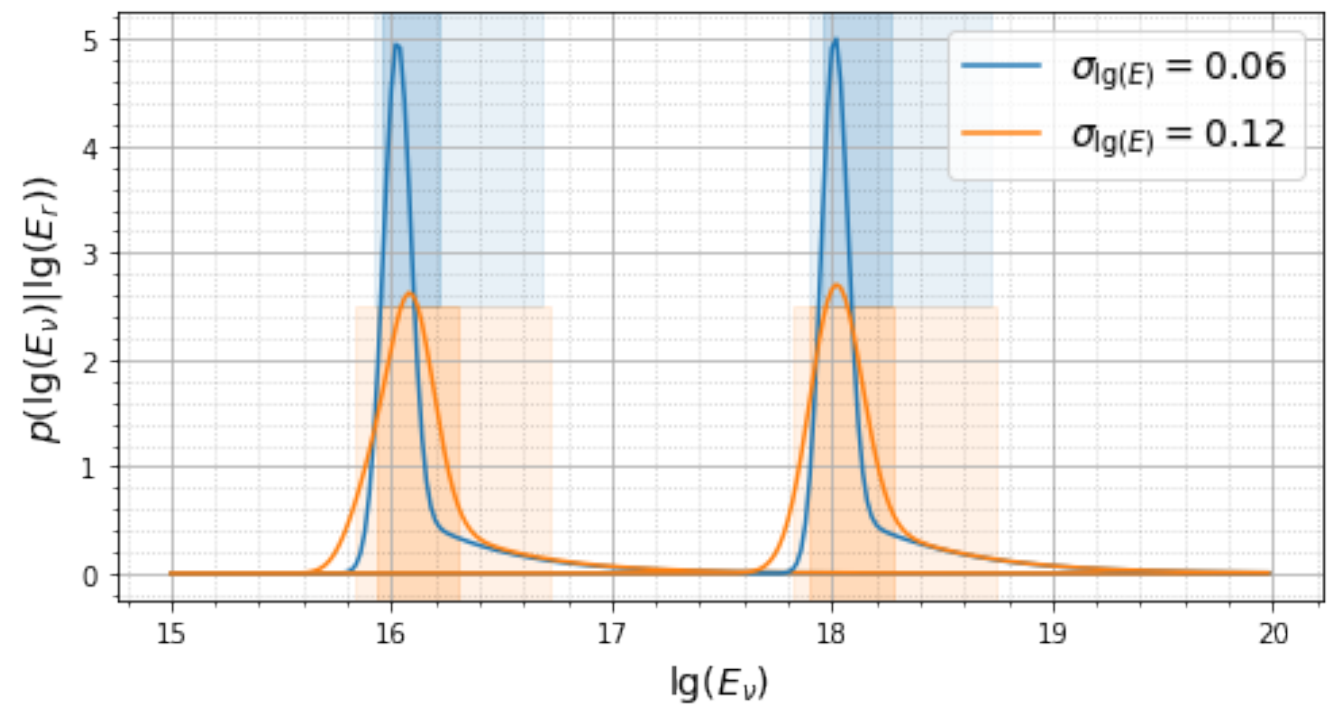

Figure 5: Comparison of the posterior distributions of the neutrino energy for reconstructed shower energies of $1 \times 10^{16} \mathrm{eV}$ and $1 \times 10^{18} \mathrm{eV}$ for uncertainties on the logarithm of the reconstructed shower energy of $12 \%$ (orange) and 6\% (blue). The shaded regions mark the $68 \%$ and $90 \%$ quantiles.

causes the $68 \%$ quantiles to change to $[15.88,16.18]$ and $[17.86,18.14]$. This happens because the tails stem from high-energy neutrinos only transferring a small fraction of their energy into the shower, a scenario that is less likely for a softer spectrum. 


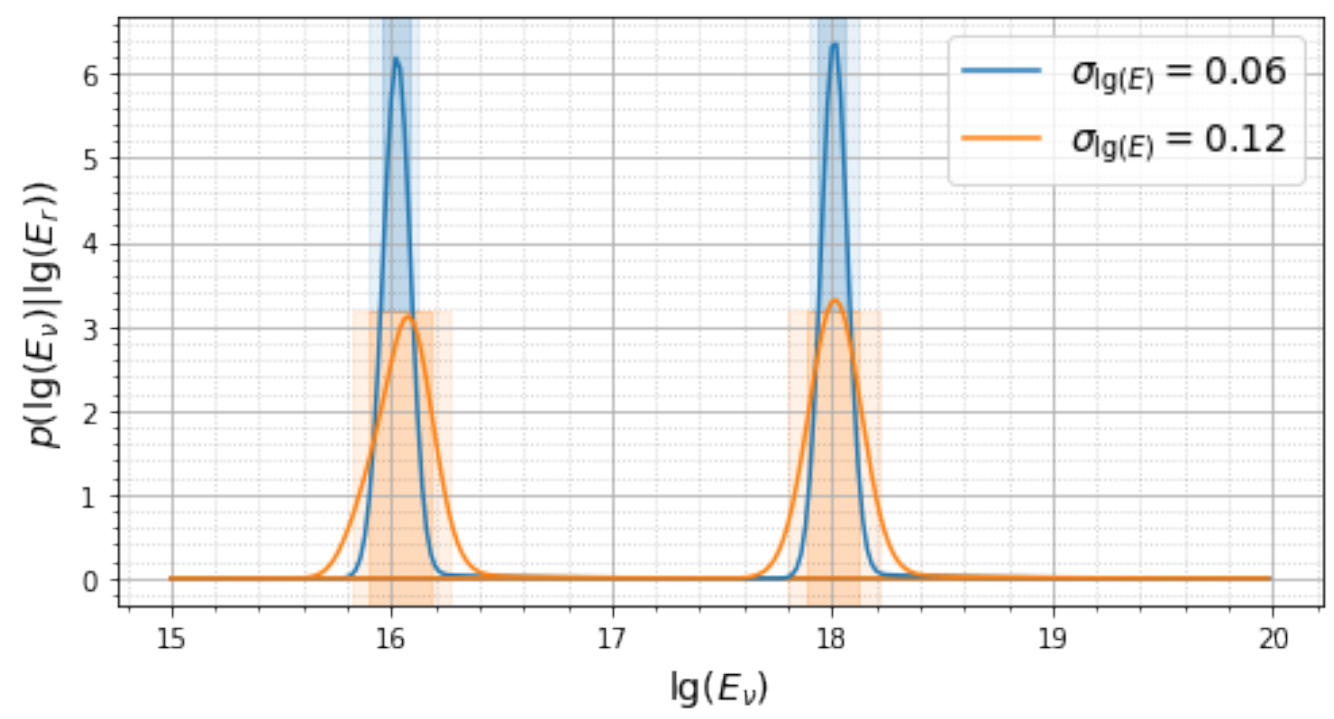

Figure 6: Comparison of the posterior distributions of the neutrino energy for reconstructed shower energies of $1 \times 10^{16} \mathrm{eV}$ and $1 \times 10^{18} \mathrm{eV}$ for uncertainties on the logarithm of the reconstructed shower energy of $12 \%$ (orange) and 6\% (blue), assuming the probability $P(e)$ for the event to be a $v_{e}+\mathrm{CC}$ event is increased to $80 \%$. The shaded regions mark the $68 \%$ and $90 \%$ quantiles.

\subsection{Shower Energy Reconstruction}

The most obvious way to try and improve the neutrino energy reconstruction is the reconstruction of the shower energy. Unfortunately, the uncertainty on $\kappa$ limits the effect this can have, as is shown in Fig. 5. Reducing the uncertainty on the logarithm of the shower energy from $12 \%$ to $6 \%$ would only reduce the size of the $68 \%$ quantile from $[15.94,16.30]$ and $[17.90,18.28]$ to $[15.96,16.22]$ and $[17.96,18.26]$ for the two examples shown. We can therefore conclude that the resolution obtained with the method discussed in Section 2.3 is already comparatively enough to not be the limiting factor.

\subsection{Flavor Identification}

The uncertainty on the neutrino energy could be reduced if events where an electron neutrino interacted via charged current interaction can be identified. So far, no method to do this has been developed, but there are ways in which it may be possible for at least some events, for example by identifying features in the spectrum of the radio signal resulting from interference between the two showers. If hints like this were identified in a specific event, it would allow us to increase the probability $P(e)$ from Eq. 4. As an example, Fig. 6 shows how the posterior probability of the neutrino energy from Fig. 5 would change if $P(e)$ is increased to $80 \%$. The tails of the distributions practically disappear and the $68 \%$ quantiles decrease to $[15.90,16.07]$ and $[17.88,18.12]$ for the $\sigma_{\lg (E)}=0.12$ case and to $[15.96,16.08]$ and $[17.94,18.06]$ for the $\sigma_{\lg (E)}=0.06$ case. These quantiles are almost equal to the shower energy uncertainty, suggesting that the shower energy resolution is exclusively the limiting factor. 


\section{Conclusion}

We have presented a method to reconstruct the energy of neutrino-induced particle showers detected by the Radio Neutrino Observatory Greenland (RNO-G) and the resolution achieved by that method. We show a model to estimate the neutrino energy based on this reconstruction and show that, while prior assumptions about the neutrino spectrum also influence the result, the neutrino energy resolution is mostly limited by uncertainties from the interaction dynamics. However, this may be overcome for some events, if flavor sensitives methods can be developed that identify the events as being caused by an electron neutrino undergoing charged current interactions.

\section{References}

[1] RNO-G collaboration, Design and Sensitivity of the Radio Neutrino Observatory in Greenland (RNO-G), JINST 16 (2021) P03025 [2010.12279].

[2] G.A. Askaryan, Excess negative charge of an electron-photon shower and the coherent radio emission from it, Zhur. Eksptl'. i Teoret. Fiz. 41 (1961) .

[3] J.V. Jelley, J.H. Fruin, N.A. Porter and T.C. Weekes, Radio pulses from extensive cosmic-ray air showers, Nature 205 (1965) 327.

[4] D. Saltzberg et al., Observation of the Askaryan Effect: Coherent Microwave Cherenkov Emission from Charge Asymmetry in High-Energy Particle Cascades, Physical Review Letters 86 (2001) 2802-2805.

[5] P.W. Gorham et al., Accelerator measurements of the Askaryan effect in rock salt: A Roadmap toward teraton underground neutrino detectors, Phys. Rev. D 72 (2005) 023002 [astro-ph/0412128].

[6] A. Cooper-Sarkar, P. Mertsch and S. Sarkar, The high energy neutrino cross-section in the Standard Model and its uncertainty, JHEP 08 (2011) 042 [1106. 3723].

[7] A. Connolly, R.S. Thorne and D. Waters, Calculation of High Energy Neutrino-Nucleon Cross Sections and Uncertainties Using the MSTW Parton Distribution Functions and Implications for Future Experiments, Phys. Rev. D 83 (2011) 113009 [1102 . 0691].

[8] R. Gandhi, C. Quigg, M.H. Reno and I. Sarcevic, Ultrahigh-energy neutrino interactions, Astropart. Phys. 5 (1996) 81 [hep-ph/9512364].

[9] C. Welling, to be submitted, Ph.D. thesis, Friedrich Alexander Universität Erlangen-Nürnberg, 2021.

[10] RNO-G collaboration, Reconstructing the neutrino energy for in-ice radio detectors, to be submitted . 


\section{Full Authors List: RNO-G Collaboration}

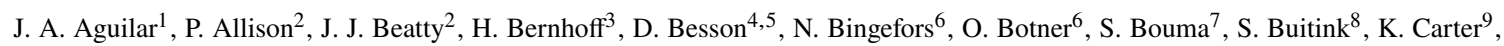
M. Cataldo ${ }^{7}$, B. A. Clark ${ }^{10}$, Z. Curtis-Ginsberg ${ }^{11}$, A. Connolly ${ }^{2}$, P. Dasgupta ${ }^{1}$, S. de Kocker ${ }^{12}$, K. D. de Vries ${ }^{12}$, C. Deaconu ${ }^{11}$, M. A. DuVernois ${ }^{13}$, C. Glaser ${ }^{6}$, A. Hallgren ${ }^{6}$, S. Hallmann ${ }^{14}$, J. C. Hanson ${ }^{15}$, B. Hendricks ${ }^{16}$, B. Hokanson-Fasig ${ }^{13}$, C. Hornhuber ${ }^{4}$, K. Hughes ${ }^{11}$, A. Karle ${ }^{13}$, J. L. Kelley ${ }^{13}$, S. R. Klein ${ }^{17}$, R. Krebs ${ }^{16}$, R. Lahmann ${ }^{7}$, U. Latif ${ }^{12}$, M. Magnuson ${ }^{4}$, T. Meures ${ }^{13}$, Z. S. Meyers ${ }^{14,7}$, K. Mulrey ${ }^{8}$, A. Nelles ${ }^{14,7}$, A. Novikov ${ }^{4}$, E. Oberla ${ }^{11}$, B. Oeyen ${ }^{18}$, H. Pandya ${ }^{8}$, I. Plaisier ${ }^{7,14}$, L. Pyras ${ }^{14,7}$, D. Ryckbosch ${ }^{18}$, O. Scholten ${ }^{12,19}$, D. Seckel ${ }^{20}$, D. Smith ${ }^{11}$, D. Southall ${ }^{11}$, J. Torres ${ }^{2}$, S. Toscano ${ }^{1}$, D. Tosi ${ }^{13}$, D. J. Van Den Broeck ${ }^{12,8}$, N. van Eijndhoven ${ }^{12}$, A. G. Vieregg ${ }^{12}$, C. Welling ${ }^{7,14}$, S. Wissel ${ }^{16,9}$, R. Young ${ }^{4}$, and A. Zink ${ }^{7}$

${ }^{1}$ Université Libre de Bruxelles, Science Faculty CP230, B-1050 Brussels, Belgium

${ }^{2}$ Dept. of Physics, Center for Cosmology and AstroParticle Physics, Ohio State University, Columbus, OH 43210, USA

${ }^{3}$ Uppsala University, Dept. of Engineering Sciences, Division of Electricity, Uppsala, SE-752 37, Sweden

${ }^{4}$ University of Kansas, Dept. of Physics and Astronomy, Lawrence, KS 66045, USA

${ }^{5}$ National Nuclear Research University MEPhI, Kashirskoe Shosse 31, 115409, Moscow, Russia

${ }^{6}$ Uppsala University, Dept. of Physics and Astronomy, Uppsala, SE-752 37, Sweden

${ }^{7}$ Erlangen Center for Astroparticle Physics (ECAP), Friedrich-Alexander-University Erlangen-Nuremberg, 91058 Erlangen, Germany

${ }^{8}$ Vrije Universiteit Brussel, Astrophysical Institute, Pleinlaan 2, 1050 Brussels, Belgium

${ }^{9}$ Physics Dept. California Polytechnic State University, San Luis Obispo CA 93407, USA

${ }^{10}$ Dept. of Physics and Astronomy, Michigan State University, East Lansing MI 48824, USA

${ }^{11}$ Dept. of Physics, Enrico Fermi Inst., Kavli Inst. for Cosmological Physics, University of Chicago, Chicago, IL 60637, USA

${ }^{12}$ Vrije Universiteit Brussel, Dienst ELEM, B-1050 Brussels, Belgium

${ }^{13}$ Wisconsin IceCube Particle Astrophysics Center (WIPAC) and Dept. of Physics, University of Wisconsin-Madison, Madison, WI 53703, USA

${ }^{14}$ DESY, Platanenallee 6, 15738 Zeuthen, Germany

${ }^{15}$ Whittier College, Whittier, CA 90602, USA

${ }^{16}$ Dept. of Physics, Dept. of Astronomy \& Astrophysics, Penn State University, University Park, PA 16801, USA

${ }^{17}$ Lawrence Berkeley National Laboratory, Berkeley, CA 94720, USA

${ }^{18}$ Ghent University, Dept. of Physics and Astronomy, B-9000 Gent, Belgium

${ }^{19}$ Kapteijn Institute, University of Groningen, Groningen, The Netherlands

${ }^{20}$ Dept. of Physics and Astronomy, University of Delaware, Newark, DE 19716, USA 